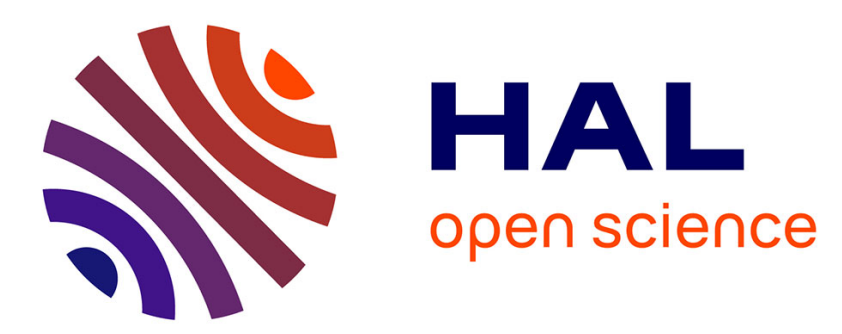

\title{
Analyse spatio-temporelle du rayonnement VHF-UHF associé à l'éclair
}

\author{
A. Bondiou, I. Taudière, Patrick Richard, F. Helloco
}

\section{To cite this version:}

A. Bondiou, I. Taudière, Patrick Richard, F. Helloco. Analyse spatio-temporelle du rayonnement VHF-UHF associé à l'éclair. Revue de Physique Appliquée, 1990, 25 (2), pp.147-157. 10.1051/rphysap:01990002502014700 . jpa-00246173

\section{HAL Id: jpa-00246173 https://hal.science/jpa-00246173}

Submitted on 1 Jan 1990

HAL is a multi-disciplinary open access archive for the deposit and dissemination of scientific research documents, whether they are published or not. The documents may come from teaching and research institutions in France or abroad, or from public or private research centers.
L'archive ouverte pluridisciplinaire HAL, est destinée au dépôt et à la diffusion de documents scientifiques de niveau recherche, publiés ou non, émanant des établissements d'enseignement et de recherche français ou étrangers, des laboratoires publics ou privés. 
Classification

Physics Abstracts

$92.90-93.85-52.90$

\title{
Analyse spatio-temporelle du rayonnement VHF-UHF associé à l'éclair
}

\author{
A. Bondiou, I. Taudière, P. Richard et F. Helloco \\ Office National d'Etudes et de Recherches Aérospatiales, BP 72, 92322 Châtillon Cedex, France
}

(Reçu le 27 février 1989, révisé le 21 juillet 1989, accepté le 18 août 1989)

\begin{abstract}
Résumé. - Les caractéristiques générales de rayonnement électromagnétique VHF-UHF de l'éclair seront examinées tant au niveau global du développement de l'orage qu'à l'échelle d'une décharge isolée. L'étude de l'évolution de l'activité électromagnétique au cours de l'orage sera confrontée à une analyse radar des paramètres de la cellule orageuse (réflectivité, dynamique). Les perspectives ouvertes par ce type d'étude seront discutées à la lumière de travaux récents décrivant les phénomènes électriques précurseurs de l'apparition de forts cisaillements de vent au voisinage du sol. Par ailleurs, l'analyse détaillée du rayonnement associé à l'éclair intranuage sera approfondie grâce à la comparaison à des expériences de laboratoire permettant de préciser les différents mécanismes mis en jeu.

Abstract. - The general features of the VHF-UHF radiation of lightning are studied both at the storm time scale and at the lightning discharge time scale. The evolution of the electromagnetic activity during the storm development is compared with the reflectivity and dynamics of the cloud given by a system of two radars. Some applications of this kind of study will be discussed in light of recent work devoted to the occurrence of thunderstorm microbursts related to the evolution of the electrical activity. In the second part of this paper, the detailed analysis of the electromagnetic radiation associated with an intracloud discharge is compared to laboratory experiments which enable to study the discharge mechanisms.
\end{abstract}

\section{Introduction.}

Si les effets directs du foudroiement d'une structure au sol ou d'un avion peuvent apparaître comme les plus redoutables, nul n'ignore cependant que le rayonnement électromagnétique lié à l'éclair peut être à l'origine de perturbations graves. En revanche, l'analyse de ce rayonnement constitue un moyen d'investigation privilégié des décharges orageuses, là où la mise en œuvre de diagnostics classiques en physique de décharges (mesures de courant, enregistrements photographiques) s'avère souvent irréalisable.

Le spectre du rayonnement associé à l'éclair s'étend de quelques $\mathrm{kHz}$ à plusieurs $\mathrm{GHz}$ et apparaît en première approximation comme inversement proportionnel à la fréquence [14]. Des mesures sélectives ont montré que la quasi-totalité des phases de l'éclair était détectable, à grande distance, dans la gamme VHF-UHF : phases de précurseurs, ou "leaders », éclairs intranuages, mécanismes secondaires liés au passage de l'arc en retour, microclaquages dispersés dans le nuage. L'étude théorique et expérimentale de la structure temporelle de ce rayonnement a fait l'objet de nombreux travaux [2, $8,10 b i s, 13]$. Ces études ont montré que le rayonnement VHF-UHF associé à l'éclair était constitué d'impulsions à front de montée de l'ordre de $5 \mathrm{~ns}$ et se répétant à un rythme de 1 à 20 impulsions par microseconde.

L'étude de décharges se propageant à la surface d'un diélectrique $[9,10]$ et présentant de nombreuses analogies avec le précurseur négatif de l'éclair, a permis de mettre en évidence le mécanisme élémentaire responsable de cette émission impulsionnelle. L'origine de cette impulsion est attribuée à la phase à fort courant, de durée inférieure à $10 \mathrm{~ns}$, réalisant la transition entre la phase de prédécharge («streamer») et le canal conducteur proprement dit (« leader »). La compréhension de ce mécanisme élémentaire constitue l'outil de base nécessaire à l'interprétation du rayonnement associé à l'ensemble du développement d'une décharge atmosphérique.

Dans cette perspective, l'ONERA a mis en œuvre, au cours de différentes campagnes in situ, un système permettant l'analyse spatio-temporelle du rayonne- 
ment VHF-UHF. Il s'agit d'un système de localisation des sources, fonctionnant à une fréquence de réception de $300 \mathrm{MHz}$ et reposant sur un principe de localisation par interférométrie. La dernière campagne d'essais, effectuée dans le sud-ouest de la France durant l'été 1984, a permis d'expérimenter une version de ce système permettant une localisation en trois dimensions, avec une précision spatiale de quelques centaines de mètres et une résolution temporelle de $1 \mu \mathrm{s}$.

Les performances de ce système, grande résolution spatio-temporelle et importantes capacités d'acquisition permettant le suivi de l'activité sur des périodes supérieures à $1 \mathrm{~h}$, ont orienté notre étude vers deux objectifs distincts :

- le premier consiste en une analyse globale de l'activité électromagnétique à l'échelle de l'orage. Cette étude a été menée en liaison avec une analyse des paramètres météorologiques de la cellule orageuse (réflectivité radar, champ tridimensionnel du vent) ;

- en second lieu, le système interférométrique permet une étude fine des caractéristiques de la décharge atmosphérique, particulièrement en ce qui concerne les éclairs intranuages. L'interprétation des données dont nous disposions nous a orientés vers des études complémentaires en laboratoire permettant de préciser les mécanismes de propagation et de rayonnement des diverses phases de la décharge.

\section{Méthode de localisation interférométrique des sources VHF-UHF.}

Le système expérimenté par l'ONERA a été développé en deux temps. Durant les campagnes COPT 81 (Côte-d'Ivoire), TRIP 82 et TRIP 83 (USA), un premier système permettant une localisation angulaire des sources (en deux dimensions) a été mis en œuvre. La détermination, en azimut et en site, de la direction de propagation de l'onde incidente est effectuée par la mesure de la différence de phase de celle-ci sur un système constitué de trois antennes distantes de $\lambda / 2$. Une mesure plus précise est réalisée en complétant ce dispositif, par un second système de trois antennes distantes de $10 \lambda$ (Fig. 1). Le détail des processus de traitement du signal ainsi que les premiers résultats obtenus ont été décrits dans de précédentes publications [17, 18]. Lors de la campagne Landes-Fronts 84 , ce système a été complété par une seconde station identique à la première et située à $9 \mathrm{~km}$ de celle-ci. Deux images angulaires d'un même événement sont ainsi obtenues de façon indépendante, chaque station étant équipée de moyens d'acquisition, de numérisation en temps réel et de stockage permettant d'atteindre une résolution temporelle de $1 \mu \mathrm{s}$. Les deux stations étant synchronisées à la $\mu \mathrm{s}$, les traitements numéri-

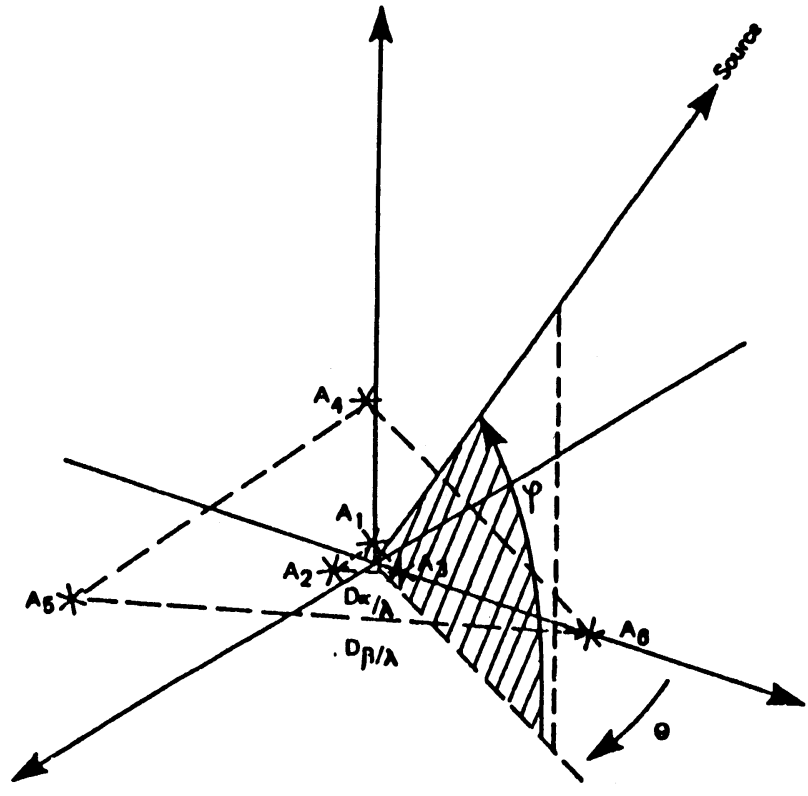

Fig. 1. - Principe de fonctionnement d'une station interférométrique. La combinaison des données fournies par le petit système (antennes $A_{1}, A_{2}, A_{3}$ ) et le grand système (antennes $A_{4}, A_{5}, A_{6}$ ) permet une localisation angulaire en azimut $\theta$ et site $\varphi$.

[Principle of interferometric location. Angular location in azimuth $\theta$ and elevation $\phi$ is obtained from data provided by the small system (antennas $A_{1}, A_{2}, A_{3}$ ) and the large one (antennas $A_{4}, A_{5}, A_{6}$ ).]

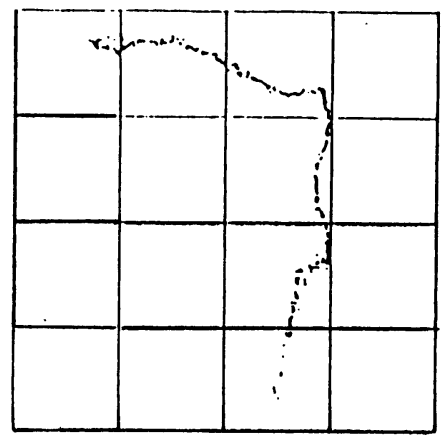

Station 1

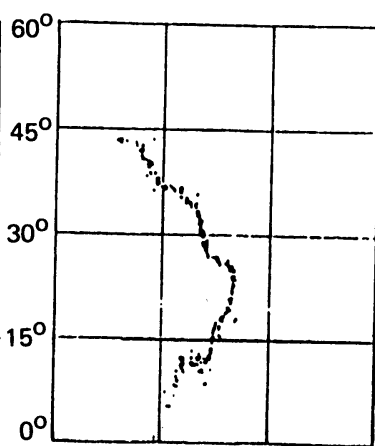

Station 2

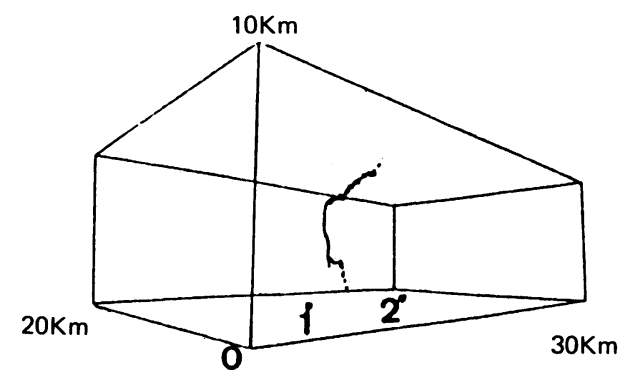

Fig. 2. - Principe de reconstruction tridimensionnelle à partir des images angulaires obtenues par chaque station. Exemple d'un événement nuage-sol.

[ 3 dimensional interferometric mapping principle using the two angular images provided by each station (example of a cloud to ground event).] 
ques en temps différé permettent ensuite une reconstruction tridimensionnelle par triangulation effectuée point par point (Fig. 2).

\section{Analyse de l'activité électromagnétique VHF-UHF à l'échelle de l'orage.}

3.1 MÉTHOdE EXPÉRIMENTALE. - Les objectifs de cette étude nécessitant l'analyse des caractéristiques des cellules orageuses, le CNET-CRPE a mis en œuvre, parallèlement à nos moyens d'analyse de l'activité électromagnétique, un système de deux radars Doppler permettant une exploration en 3 dimensions du milieu nuageux. Lors de l'expérience Landes-Fronts 1984, ces deux radars, disposés comme l'indique la figure 3 , ont permis la mesure de la réflectivité et du champ de vent des cellules orageuses, selon des pixels de $1 \mathrm{~km} \times 1 \mathrm{~km} \times 1 \mathrm{~km}$. Chaque exploration radar, effectuée par plans successifs d'élévation croissante, dure environ $4 \mathrm{~min}$. Afin de s'adapter à ces conditions particulières d'analyse, les données de localisation interférométrique ont été traitées suivant des pixels identiques. Compte tenu de la masse d'informations à traiter (analyse de plus d'une heure d'activité), les données de localisation ont également été moyennées sur des intervalles de $100 \mu \mathrm{s}$ afin de faciliter les traitements numériques. Les localisations présentées dans ce paragraphe seront ainsi des barycentres des sources observées dans chaque intervalle de $100 \mu$ s et cumulées par pixels de $1 \mathrm{~km}$ de côté.

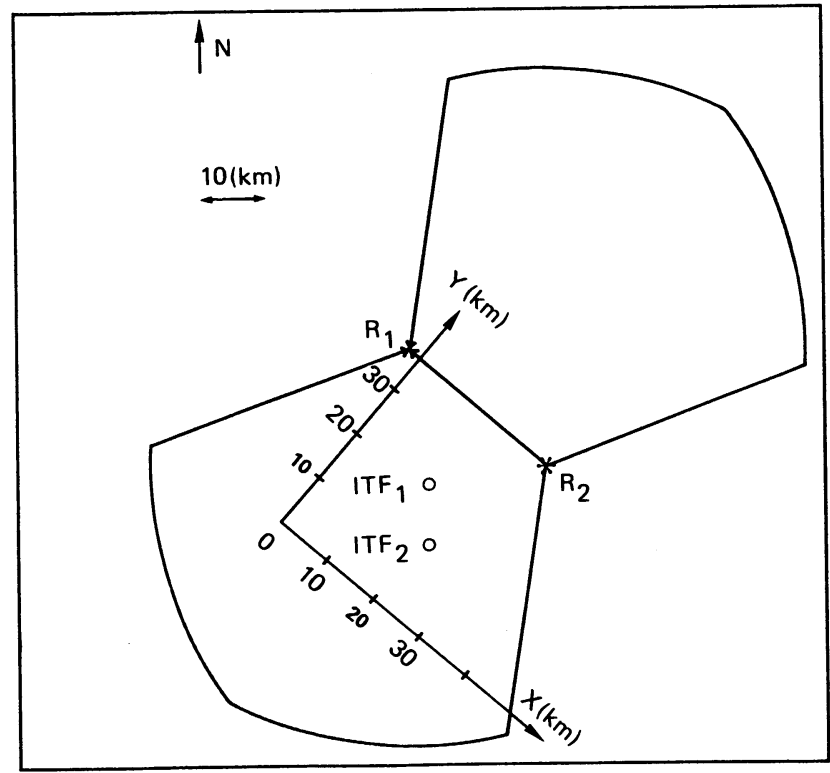

Fig. 3. - Expérience Landes-Fronts 84. Situation des stations interférométriques (ITF1 et IFT2) et des deux radars du CRPE $\left(R_{1}\right.$ et $\left.R_{2}\right)$.

[Landes-Fronts 84 experiment. Location of the two interferometric stations (ITF1 and IFT2) and of the two CRPE' radars $\left(R_{1}\right.$ and $\left.R_{2}\right)$.]
Les résultats présentés ici concernent l'orage du 20 juin 1984, entre $20: 30$ et $21: 44$ TU, où apparaissent clairement les diverses étapes du développement de la cellule orageuse et où l'on a pu mettre en évidence l'évolution spécifique de l'activité électromagnétique associée aux décharges.

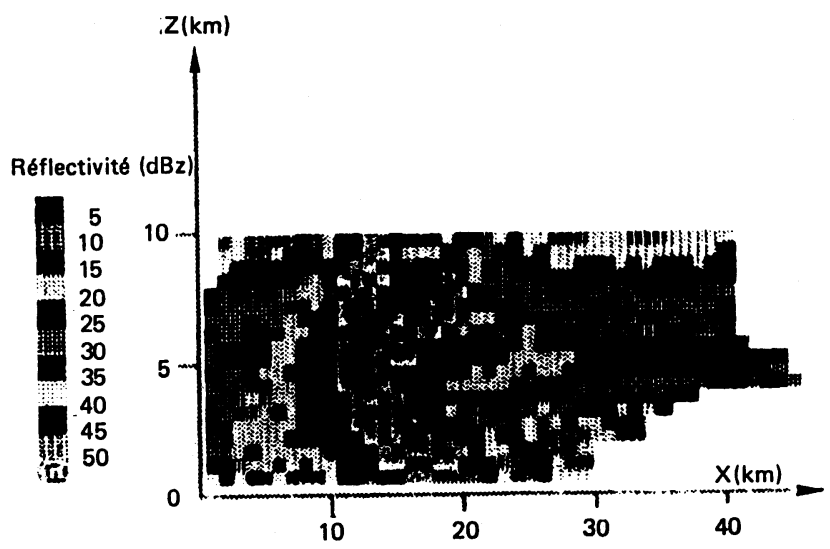

a)

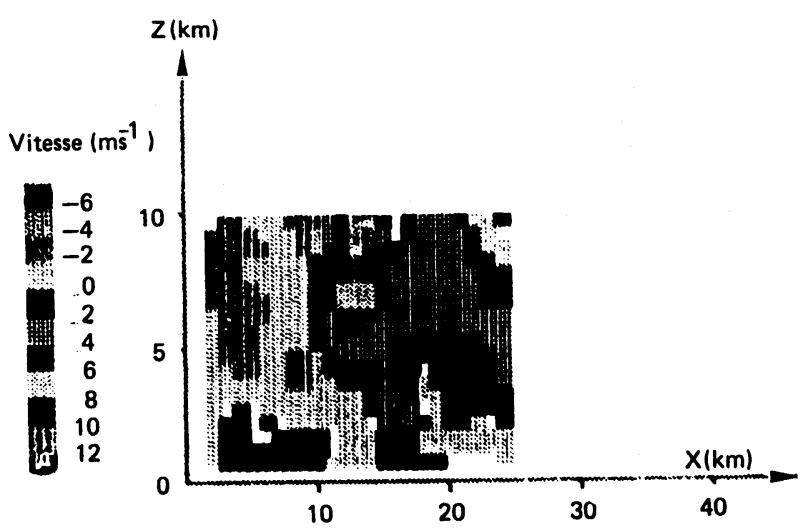

b)

Fig. 4. - (a) Coupe verticale de la réflectivité du milieu nuageux exprimée en dBz. Orage du 20/6/84, $20: 44$ TU. (b) Représentation, selon la même coupe verticale, de la composante verticale du vent. 20/6/84, $20: 44$ TU.

[(a) Vertical cross section of the reflectivity of the cloud (in dBz). Storm of June 20, 1984, $20: 44$ TU. (b) Vertical cross section of the vertical velocity of the wind. Storm of June 20, 1984, $20: 44$ TU.]

3.2 RÉSUltATS. - La première demi-heure de l'orage se traduit par le développement d'un système fortement convectif se structurant vers $20: 45 \mathrm{TU}$ selon deux cellules distinctes caractérisées par de fortes ascendances (Figs. $4 \mathrm{a}$ et $4 \mathrm{~b}$ ). L'évolution du système à partir de 21:00 TU fait, par contre, apparaître progressivement une structure dite «stratiforme » caractéristique de la phase de dissipation des cellules orageuses (Fig. 5a). La figure $5 \mathrm{~b}$ indique 


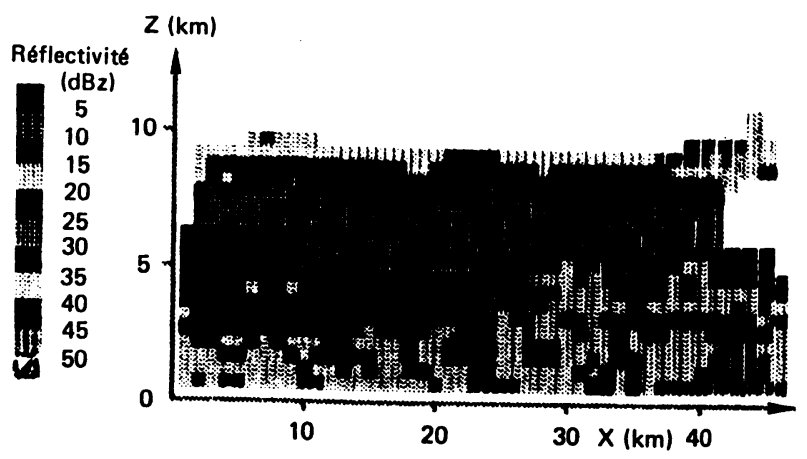

a)

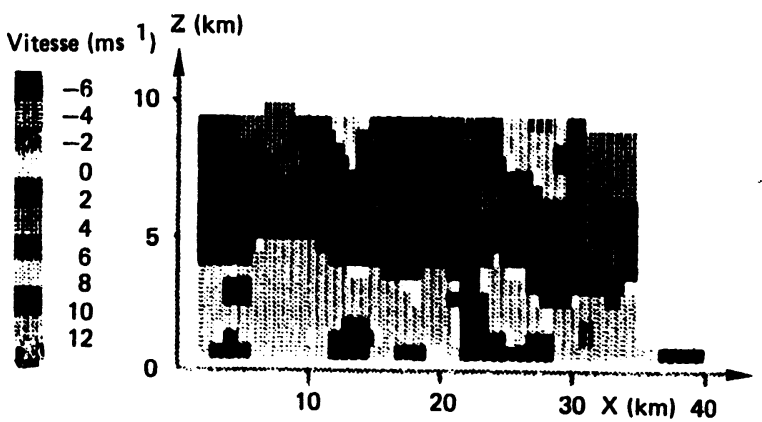

b)

Fig. 5. - (a) Coupe verticale de la réflectivité du milieu nuageux. Système stratiforme. 20/6/84, 21:15 TU. (b) Représentation, selon la même coupe verticale, de la composante verticale du vent. 20/6/84, 21 : 15 TU.

[(a) Vertical cross section of the reflectivity in the stratified system $(20 / 6 / 84,21: 15 \mathrm{TU})$. (b) Vertical cross section of the vertical velocity of the wind (20/6/84, 21 : $15 \mathrm{TU})$.]

de très faibles valeurs de vent vertical dans cette seconde séquence.

L'activité électromagnétique correspondant à la première phase est constituée de maxima locaux, d'une durée de vie de quelques minutes, coïncidant avec les zones de plus forte réflectivité (Figs. 6a et 6b). L'intensité de l'activité électromagnétique peut être évaluée à partir du nombre de sources détectées dans un intervalle de temps donné. Une source élémentaire de rayonnement correspond ici à la localisation, moyennée sur $100 \mu \mathrm{s}$ des éléments rayonnants détectés par le système. Durant la phase convective, l'activité électromagnétique est intense (6 900 sources détectées en $30 \mathrm{~min}$ ), continue temporellement et correspondant essentiellement à des décharges intranuages distribuées autour d'une altitude moyenne de $5 \mathrm{~km}$.

Ces caractéristiques contrastent fortement avec celles correspondant à la seconde séquence, c'est-àdire à la structure stratiforme. En effet, l'activité électromagnétique observée à partir de 21:00 TU est plus faible (1 190 sources détectées en $45 \mathrm{~min}$ ) et apparaît de façon discontinue, les périodes d'activité étant séparées par des intervalles de 1 à $2 \mathrm{~min}$. Par

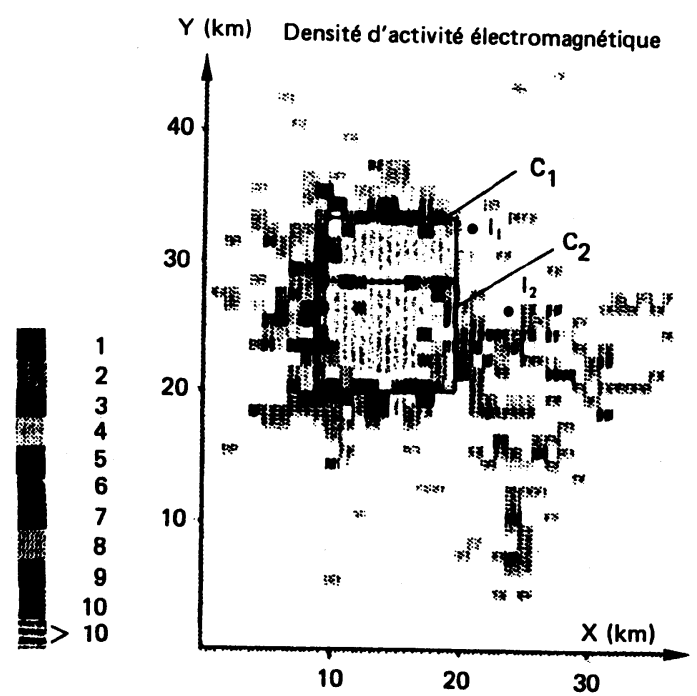

a)

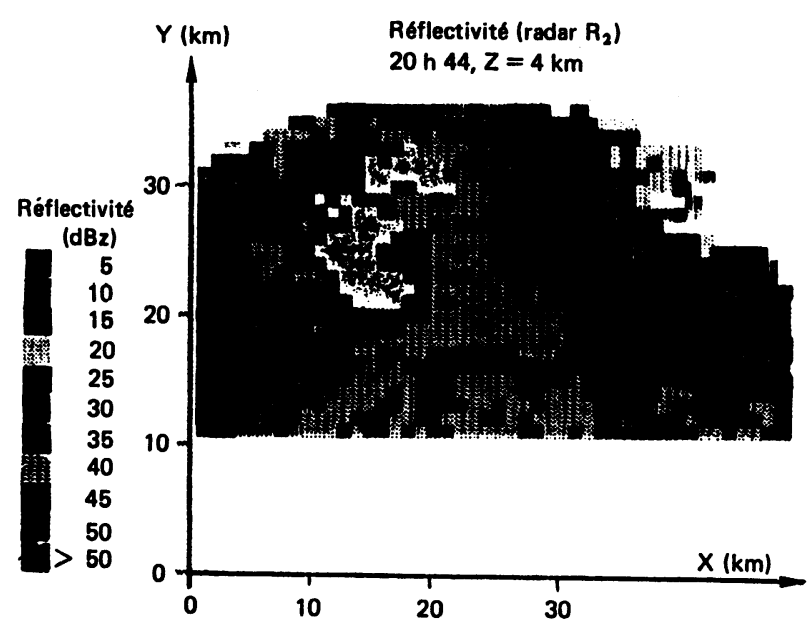

b)

Fig. 6. - (a) Projection horizontale du taux d'activité électromagnétique, selon les pixels de $1 \mathrm{~km} \times 1 \mathrm{~km}$ (de $20: 45$ à 20:50 TU). (b) Coupe horizontale (à $4 \mathrm{~km}$ d'altitude) de la réflectivité du milieu nuageux (exploration radar de $20: 44 \mathrm{TU}$ ).

[(a) Horizontal projection of the lightning activity rate in pixels of $1 \mathrm{~km} \times 1 \mathrm{~km}$ (number of $100 \mu \mathrm{s}$, sources observed during a $5 \mathrm{~mm}$ interval from $20: 45$ to $20: 50 \mathrm{TU}$ ). (b) Horizontal cross section (at $Z=4 \mathrm{~km}$ ) of the cloud reflectivity (20:44 TU).]

ailleurs, la proportion de sources localisées entre 0 et $2 \mathrm{~km}$ est en forte augmentation par rapport à la première phase, ce qui semble indiquer un accroissement du taux d'événements nuage-sol (jusqu'à $20 \%$ de l'activité totale).

3.3 Discussion. - La corrélation des données radar et des mesures interférométriques indique que le maximum d'activité électrique est localisé à l'intérieur des zones de plus forte réflectivité $(\geqslant 50 \mathrm{dBz})$, caractérisées par de fortes ascendances $(\geqslant 15 \mathrm{~m} / \mathrm{s})$. 
Lors de la phase de forte convection, l'activité électromagnétique maximale est située à $5 \mathrm{~km}$ d'altitude: on peut penser, d'après des observations similaires effectuées par Lhermitte et Williams [11], que cette altitude préférentielle correspond au centre du dipôle constitué par la zone de charges positives et la zone de charges négatives formant la structure électrique de base du nuage. L'existence d'une activité électrique persistant pendant la phase stratiforme est encore mal expliquée, la structure de champ électrique associée étant probablement moins favorable à l'initiation des décharges.

Les corrélations effectuées ici entre une analyse radar du milieu et l'étude de l'activité électromagnétique semblent être promises à de nouvelles applications. En effet, Williams [23] a mis en évidence les liens existant entre l'évolution du taux d'activité intranuage (par opposition à l'activité nuage-sol) et l'apparition de forts cisaillements de vent au voisinage du sol («windshear»). Selon cet auteur, leur occurrence suivrait, quasi invariablement, à 7 et 8 minutes d'intervalle, le maximum d'activité intranuage (Fig. 7).

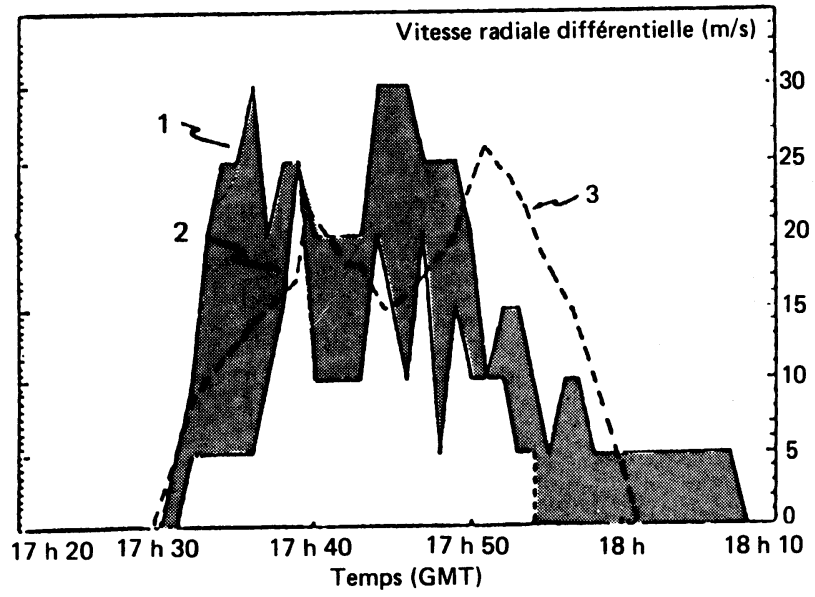

Fig. 7. - Evolution comparée du taux d'activité totale, intranuage et nuage-sol (courbe 1), du taux d'activité nuage-sol (courbe 2) et de la vitesse différentielle du vent au voisinage du sol (courbe 3 ). Les maxima de la courbe 3 indiquent l'apparition du phénomène de cisaillement, succédant dans la quasi-totalité des cas au maximum d'activité intranuage, dans un délai de 7 à $8 \mathrm{~min}$ (d'après Williams, 1988).

[Simultaneous variations of the total activity rate (intracloud and cloud to ground-curve (1)), the cloud to ground rate (-curve (2)) and the differential outflow velocity near the ground (-curve (3)). Maxima of curve (3) indicate the occurrence of microburst, 7-8 min delayed from the maximum rate of intracloud activity (from E. Williams, 1988).]

Ce maximum, coïncidant avec le développement vertical maximal de la cellule, serait suivi d'une décroissance de l'activité totale et d'une augmenta- tion parallèle du taux de coups au sol. Certaines de ces remarques, confirmées par nos propres observations, semblent ouvrir la voie à de nouveaux modes de détection des «windshear» qui constituent un des risques majeurs d'accidents d'avion en période orageuse.

Ces résultats, encore très récents, doivent cependant être confirmés et approfondis par de nouvelles expériences associant l'étude du milieu nuageux et la détection de l'activité électromagnétique.

\section{Analyse du rayonnement électromagnétique à l'échelle de la décharge}

Compte tenu des objectifs de cette étude, nous avons utilisé ici les capacités maximales de résolution spatiale et temporelle du système : ce dernier fonctionne ainsi comme un véritable système d'imagerie des décharges. La reconstruction tridimensionnelle des sources VHF-UHF associées aux décharges permet d'en déterminer les principales caractéristiques : vitesses de propagations, longueurs. Par ailleurs, des représentations en deux dimensions (projections de la sphère céleste) seront également utilisées dans la mesure où elles fournissent suffisamment d'informations pour une analyse qualitative des phénomènes.

4.1 DESCRIPTION GÉNÉRALE DE L'ORGANISATION DES SOURCES. - Les images $8 \mathrm{a}$ et $8 \mathrm{~b}$ représentent en 3 dimensions les sources localisées pendant deux périodes successives de 7 minutes (orage du 20/06/84 de $20: 31$ à $20: 45 \mathrm{TU}$ ). Les sources observées sont soit organisées en filaments longs de plusieurs kilomètres, soit isolées et dispersées dans le milieu nuageux. Il semble que 80 à $90 \%$ de l'activité soit localisée à l'intérieur du nuage : il s'agit des phases de l'éclair traditionnellement les plus mal connues, du fait de leur accès expérimental particulièrement malaisé. Nous nous attacherons donc plus précisément à l'étude des mécanismes de la décharge intranuage, cette analyse étant rendue possible par le système interférométrique.

Les figures 9 et 10 présentent deux exemples caractéristiques des événéments observés lors du développement d'une décharge intranuage. Celui-ci peut être décomposé en deux phases :

- la première phase, d'une durée de quelques centaines de ms, est caractérisée par des sources dispersées, apparaissant à un rythme croissant de quelques sources/ms jusqu'à 1 source/ $\mu$ s. Malgré la structure «dendritique » caractéristique de cette première phase, on peut déterminer une direction générale de déplacement du phénomène (Figs. 9a et 10a). Il est également possible de distinguer des sources regroupées en filaments pour lesquels il sera possible de calculer une vitesse de propagation. La durée de cette première phase, ainsi que les rythmes 


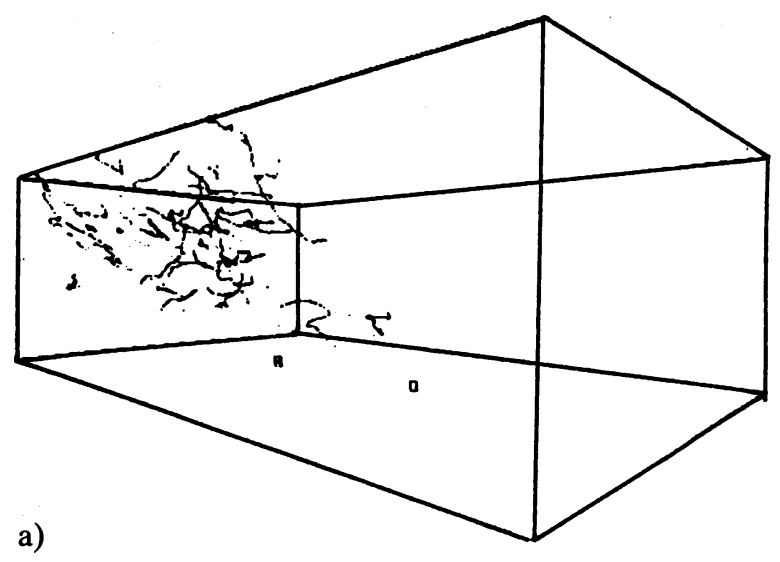

a)

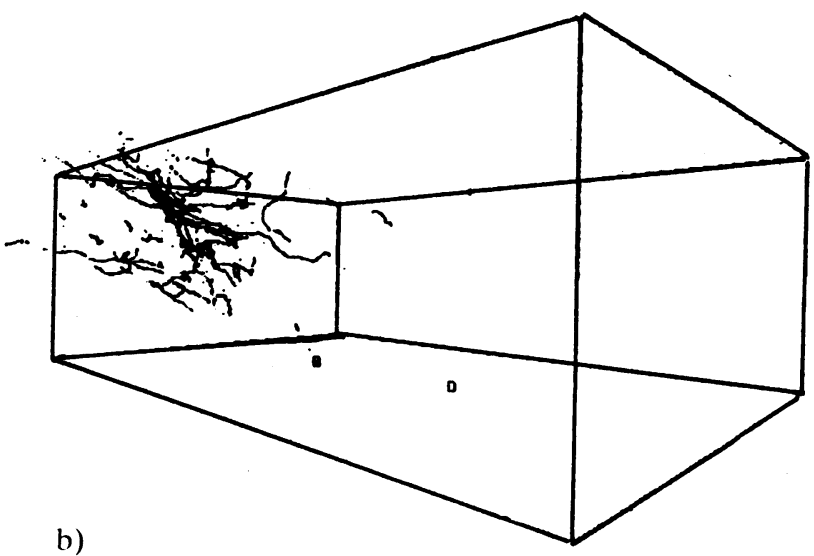

Fig. 8. - Représentation 3D des sources observées pendant 2 intervalles consécutifs de $7 \mathrm{~min}$ (orage du $20 / 6 / 84,20: 31 \rightarrow 20: 38$ et $20: 38 \rightarrow 20: 45$ TU).

[3D mapping of VHF sources located during two consecutive 7 min time intervals $(20 / 6 / 84,20: 31$ to $20: 38 \mathrm{TU}$ and $20: 38$ to $20: 45$ TU).]

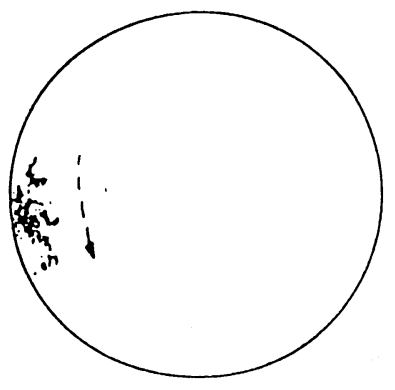

a)

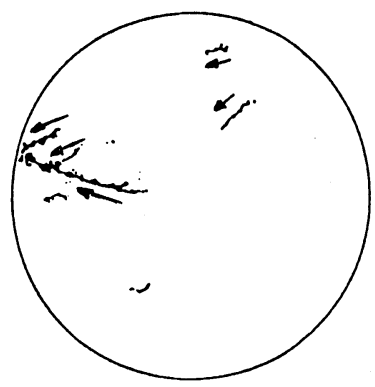

b)
Fig. 9. - (a) Projection des sources observées pendant la $1^{\text {rc }}$ phase de développement d'un intranuage (durée $400 \mathrm{~ms}$ ). (b) Projection des sources observées pendant la $2^{\mathrm{c}}$ phase (durée $400 \mathrm{~ms}$ ).

[2D mapping of VHF sources observed during the first phase of an intracloud flash development $(400 \mathrm{~ms}$ duration). (b) 2D mapping of VHF sources observed during the second phase of the same intracloud flash development (400 ms duration).]

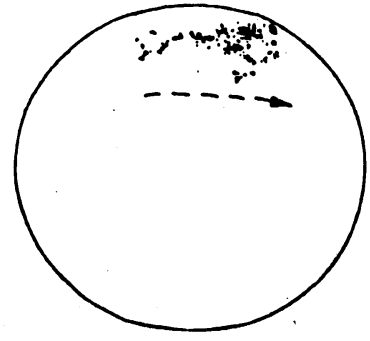

a)

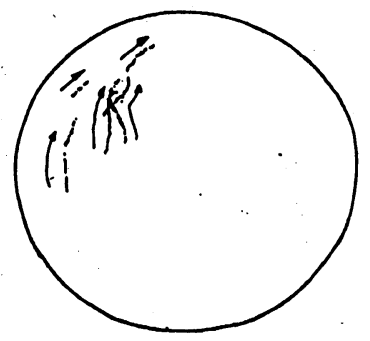

b)
Fig. 10. - (a) Projection des sources observées pendant la $1^{\text {rc }}$ phase de développement d'un intranuage (durée $500 \mathrm{~ms}$ ). (b) Projection des sources observées pendant la $2^{c}$ phase (durée $300 \mathrm{~ms}$ ).

[(a) First phase of an other intracloud flash $(500 \mathrm{~ms}$ duration). (b) Second phase of the intracloud flash of figure 10 (a) (300 ms duration).]

d'émission qui la caractérisent, semblent indiquer qu'elle se compose de processus de microclaquages dans le nuage ("preliminary breakdown ») et qu'elle inclut également la phase classique de développement de précurseurs (ou « leaders »);

- les dernières centaines de ms de l'activité intranuage sont constituées d'une succession de périodes d'activité de 50 à $500 \mu$ s où la cadence d'émission atteint 1 source/ $\mu$ s (ce qui constitue, rappelons-le, la limite de résolution du système d'acquisition). L'intervalle de temps entre deux périodes d'activité consécutives est de l'ordre de $10 \mathrm{~ms}$. Les sources sont organisées en filaments de quelques $\mathrm{km}$ de long, convergeant vers la zone d'activité initiale (Figs. 9b et 10b). Les vitesses de propagation correspondantes sont distribuées autour d'une valeur moyenne de $2 \times 10^{7} \mathrm{~ms}^{-1}$.

La morphologie générale de cette seconde phase l'identifie aux processus intranuages observés par divers auteurs et dénommés, selon les cas, « recoil streamers », « K-changes » ou « Q-noise » $[3,5,15$, 19]. On admet communément, faute d'investigations plus approfondies, que ces phénomènes sont l'équivalent intranuage du processus d'arc en retour.

\subsection{ANALYSE QUANTITATIVE DES PARAMÈTRES DE} PROPAGATION. - L'histogramme de la figure 11 présente la distribution des vitesses de propagation calculées sur plus de 33000 sources. Il est possible d'effectuer une classification plus précise des phénomènes en utilisant la mesure de leur rythme d'émission, i.e., l'intervalle de temps séparant deux sources consécutives, moyenné sur la durée d'une propagation.

La figure 12 indique en effet que l'histogramme des vitesses est en fait constitué de deux classes : la première, correspondant à un rythme d'émission de 1 source/ $\mu \mathrm{s}$, est centrée sur une vitesse de $2 \times 10^{7} \mathrm{~ms}^{-1}$ alors que la seconde, qui comprend 


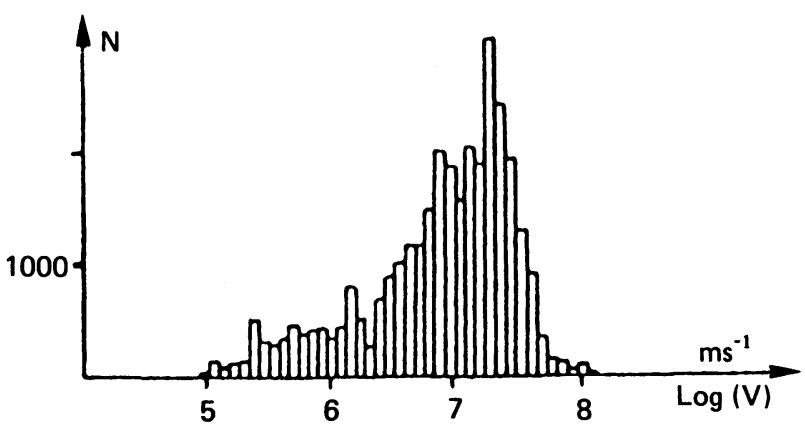

Fig. 11. - Histogramme des vitesses de propagation. $N$ : nombre de sources (total $=33000$ sources).

[Histogram of propagation velocities. $N$ : number of sources (total number $=33000)$.]

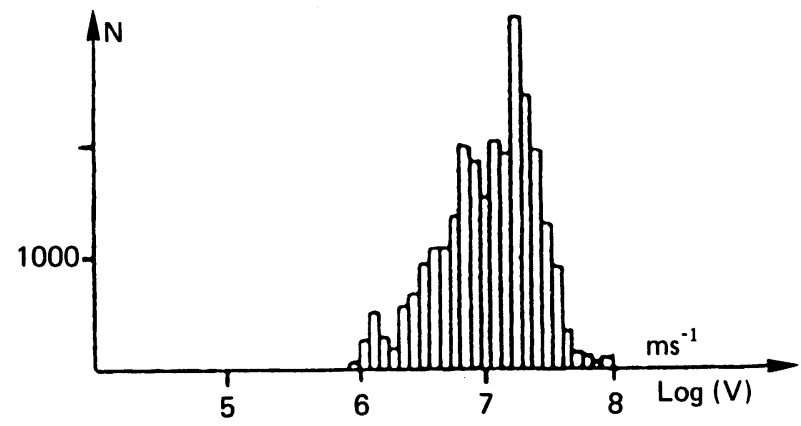

a)

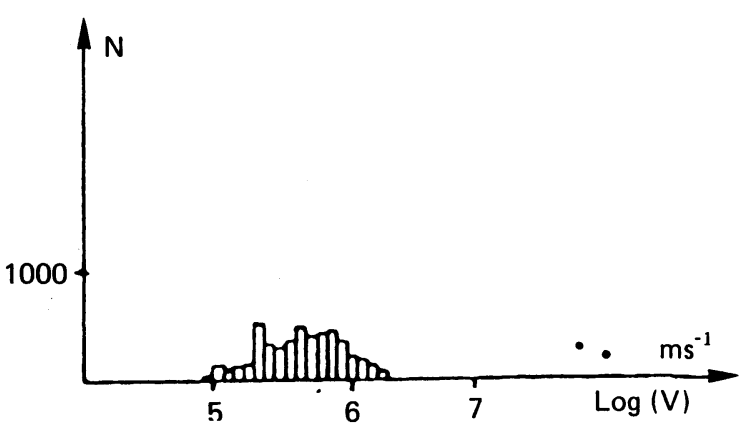

b)

Fig. 12. - (a) Décomposition de l'histogramme de la figure 11 selon le rythme d'émission. Phénomènes de rythme d'émission supérieur ou égal à $1 \mu \mathrm{s}$. (b) Décomposition de l'histogramme de la figure 11 selon le rythme d'émission. Rythme d'émission entre $1 / 30 \mu$ s et $1 / 2 \mu$ s.

[Histograms of propagation velocities on phenomena : with an emission rate higher or equal to $1 / 1 \mu \mathrm{s}$; with lower emission rate (from $1 / 30 \mu$ s to $1 / 2 \mu s$ ).]

toutes les propagations associées à un rythme d'émission plus faible, s'étend de $10^{5} \mathrm{~ms}^{-1}$ à quelque $10^{6} \mathrm{~ms}^{-1}$. La première classe correspond à l'évidence aux « recoil streamers» décrits au paragraphe 3.1. On peut remarquer d'ores et déjà que les propagations appartenant à la seconde classe $\left(10^{5}<v<\right.$ quelque $\left.10^{6} \mathrm{~ms}^{-1}\right)$, observées durant la première phase de développement de l'éclair intranuage, sont caractérisées par des vitesses voisines de celles classiquement attribuées à la phase «leader » de l'éclair [22]. Une analyse détaillée des caractéristiques d'un tel événement est présentée figures 13a à 13d. Dans la grande majorité de leaders étudiés, on constate que la distance moyenne entre deux sources consécutives est de l'ordre de $30 \mathrm{~m}$ et que le rythme d'émission se situe à environ 1 source $/ 10 \mu \mathrm{s}$. La périodicité spatiale mesurée ici est en accord avec les mesures effectuées par Proctor à partir de l'émission radioélectrique d'un précurseur par bonds ou « stepped-leader » [16].

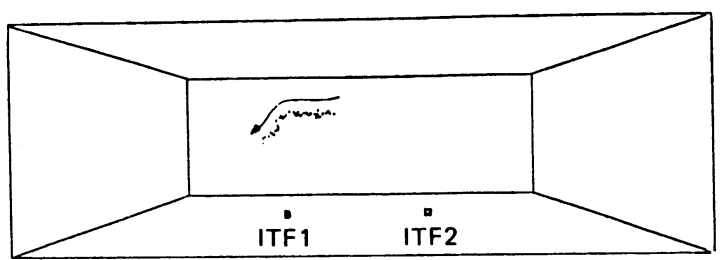

a)

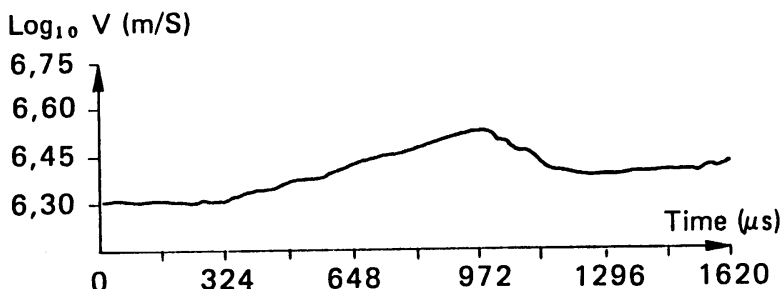

b)

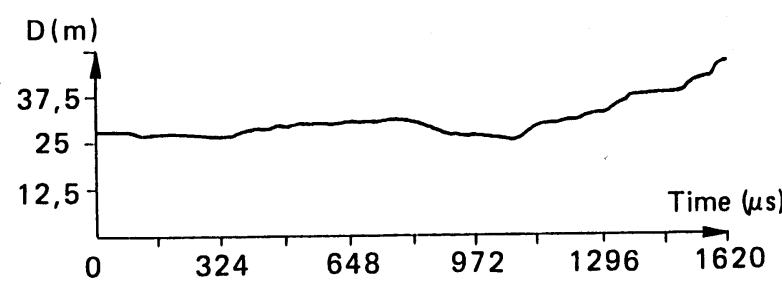

c)

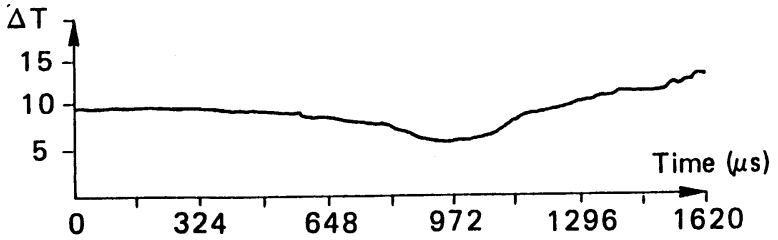

d)

Fig. 13. - (a) Représentation 3D de la propagation d'un leader intranuage (durée $1,6 \mathrm{~ms}$, longueur totale : $3,6 \mathrm{~km}$ ). (b, c, d) Evolution temporelle, au cours de la propagation du leader, des paramètres suivants: $\log V$, $V$ : vitesse de propagation; $D:$ distance entre deux sources consécutives; $\Delta T$ : intervalle de temps entre deux sources consécutives (en $\mu \mathrm{s})$.

[Temporal evolution, during the leader propagation of the following parameters : $\log V:(V:$ propagation velocity), $D$ : distance between two consecutive sources, $\Delta T$ : time interval between two consecutive sources (in $\mu \mathrm{s})$.] 
4.3 DISCUSSION : COMPARAISON DES RÉSULTATS AVEC LES MESURES EFFECTUÉES SUR DES DÉCHARGES DE LABORATOIRE. - L'étude de décharges de laboratoire offre la possibilité d'effectuer des mesures de champ rayonné par des phénomènes dont les mécanismes sont relativement bien connus. Cette étude a été entreprise dans le cas de leaders de $8 \mathrm{~m}$ de long, dont les caractéristiques générales sont voisines de celles de l'éclair naturel.

\section{Mesures effectuées en laboratoire.}

Cette étude a été réalisée au Laboratoire d'Essais Haute Tension de l'EDF (Centre des Renardières) où les mécanismes conduisant au claquage sur de grands intervalles d'air sont étudiés depuis de nombreuses années (Groupe des Renardières [4]). Notre contribution à ces études consiste en l'analyse du rayonnement électromagnétique émis par des leaders des deux polarités, générés dans un intervalle pointe-plan de $8 \mathrm{~m}$, grâce à un générateur Marx de $6 \mathrm{MV}$. Une vue générale du circuit d'essais est donnée figure 14. Les principaux moyens expérimentaux mis en œuvre sont les suivants :

- mesure du courant à la pointe haute tension grâce à un shunt de $146 \mathrm{~m} \Omega$;

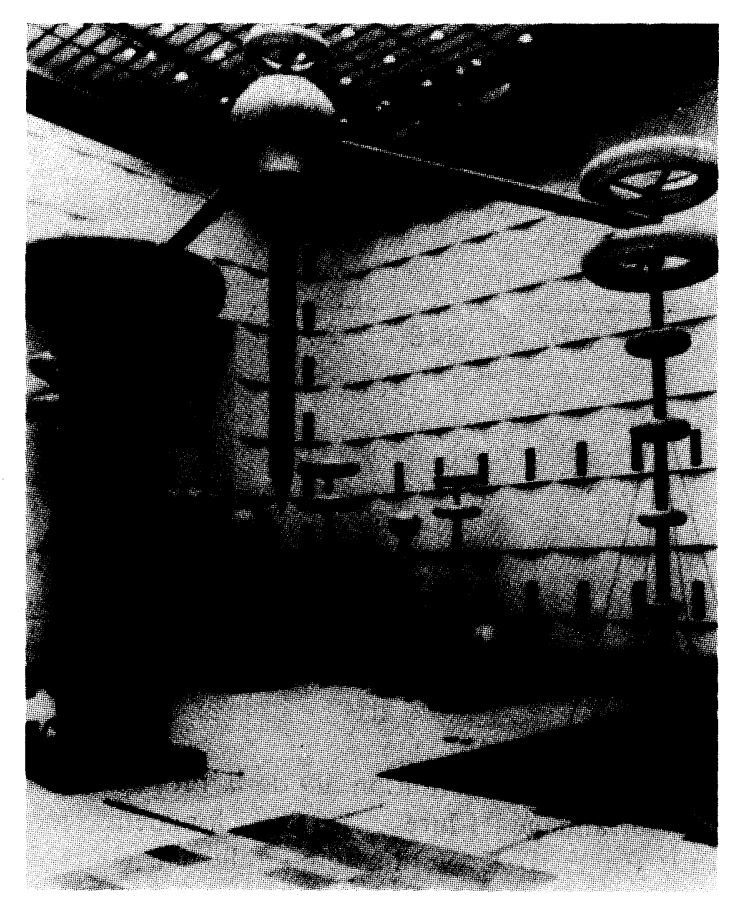

Fig. 14. - Vue générale du circuit d'essais. A gauche, le générateur Marx $6 \mathrm{MV}$; au centre, l'intervalle pointeplan; à droite : le diviseur capacitif permettant la mesure de la tension de la pointe.

[General view of the high voltage apparatus. From left to right : Marx generator (6 MV) ; rod-plane gap ; capacitive divider for voltage measurements.]
- mesure du champ rayonné à $15 \mathrm{~m}$ de la décharge grâce à un capteur de bande passante $30-100 \mathrm{MHz}$;

- enregistrements de caméragrammes à balayage temporel (mode «streak»).

Les mesures sont transmises par fibres optiques à des numériseurs rapides Tektronix RTD 710 (période d'échantillonnage : $10 \mathrm{~ns}$ ).

\subsection{ETUdE DU LEADER EN POLARITÉ POSITIVE. -} Les caméragrammes à balayage temporel (Fig. 15a) indiquent que la propagation du précurseur positif est essentiellement continue bien que de brusques réilluminations puissent intervenir en cours de développement. La vitesse moyenne de propagation est de l'ordre de $2 \times 10^{4} \mathrm{~ms}^{-1}$; le courant mesuré à la pointe ne dépasse pas quelques ampères, même lors d'une réillumination (Fig. 15b). Celles-ci, dont l'occurrence semble liée au taux d'humidité ambiant, sont associées à une faible émission VHF ( $\frac{\mathrm{d} E}{\mathrm{~d} t} \leqslant$ $1,5 \times 10^{9} \mathrm{~V} \cdot \mathrm{m}^{-1} \mathrm{~s}^{-1}$ à $\left.15 \mathrm{~m}\right)$ tandis que la phase de propagation continue ne donne lieu à aucune émission détectable (Fig. 15c).

\subsection{ETUDE DU LEADER EN POLARITÉ NÉGATIVE. -} La progression du leader négatif est discontinue, s'effectuant par bonds successifs (« steps ») associés à une brutale réillumination du canal. Typiquement, une décharge d'une dizaine de mètres inclut 3 ou 4 « steps » avant la phase d'arc qui suit la jonction du précurseur au sol. A chaque bond, la décharge progresse d'une longueur égale à la dimension du panache de streamers situé en tête de la décharge (Fig. 16a). Le courant mesuré à la pointe, voisin de zéro entre 2 steps, atteint 300 à 400 A lors de chaque step : les mesures, très reproductibles, indiquent que l'impulsion de courant est constituée de deux pics, la durée du premier front de montée n'excédant pas 30 ns (Fig. 16b).

Le rayonnement VHF associé consiste en une forte émission impulsionnelle associée à chaque bond : le champ rayonné apparaît sous la forme de trains d'impulsions, d'une durée totale de $5 \mu \mathrm{s}$ environ (Fig. 16c). Le temps de montée $10 \%-90 \%$ de la première impulsion est inférieur à $20 \mathrm{~ns}$ et il semble que les impulsions suivantes soient liées à des oscillations du circuit d'essais et à des réflexions sur les parois métalliques du laboratoire $[20,6]$.

Les valeurs crêtes de $\frac{\mathrm{d} E}{\mathrm{~d} t}$ associées à chaque step sont en moyenne supérieures de $30 \mathrm{~dB}$ aux impulsions observées lors des réilluminations du leader positif.

5.3 DISCUSSION : INTERPRÉTATION DES DONNÉES INTERFÉROMÉTRIQUES. - Les mesures de laboratoire ont mis en évidence les différences majeures existant, du point de vue électromagnétique, entre les leaders des deux polarités. Sans qu'il soit possible 


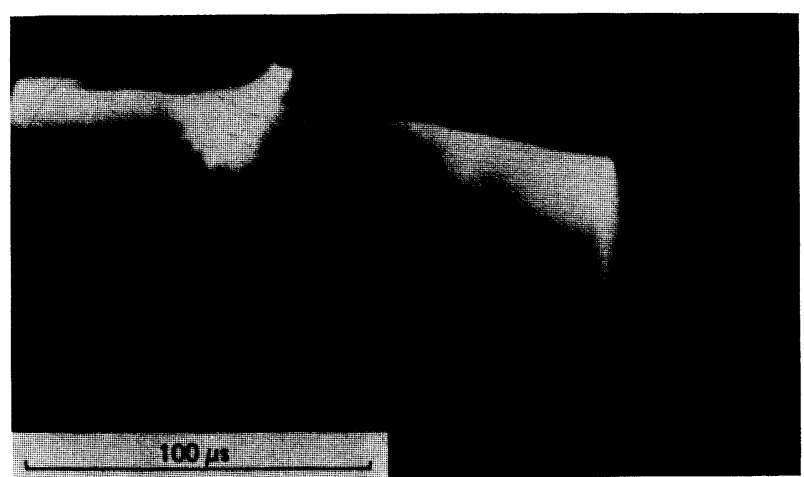

a)

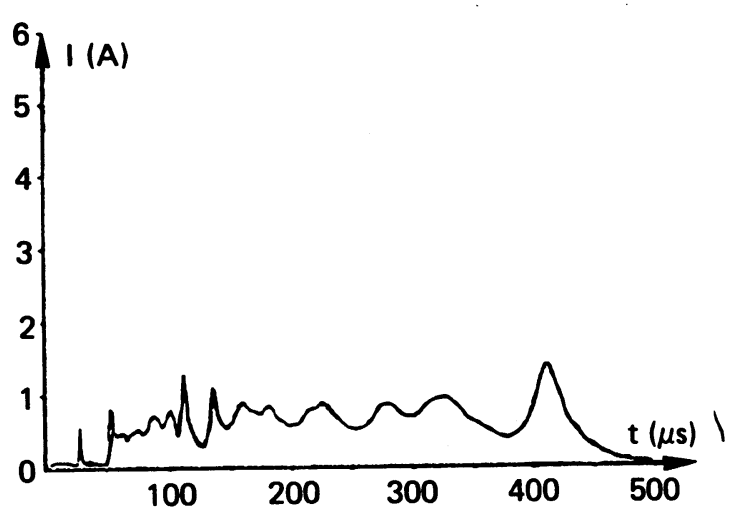

b)

$V m^{-1} s^{-1}$
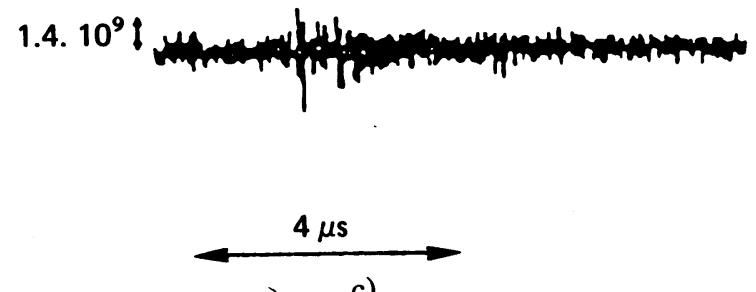

c)

Fig. 15. - (a) Caméragramme à balayage temporel montrant le développement d'un leader positif. Une réillumination intervient au temps $t \simeq 80 \mu$ s (intervalle de $8 \mathrm{~m}$, tension : 2,4 MV). (b) Enregistrement de courant associé au leader positif (d'après le Groupe des Renardières, 1977). (c) Enregistrement de la dérivée du champ rayonné associé à une réillumination du leader positif.

[(a) Streak cameragram of a positive leader development. A restrike can be observe at time $t=80 \mu$ s (gap length : $8 \mathrm{~m}$; voltage : $2.4 \mathrm{MV}$ ). (b) Current variations associated with a positive leader. (c) Recording of the radiative $E$ field derivative associated with a restrike.]

d'interpréter de façon exhaustive les résultats présentés ici, il semble que la décharge de polarité négative est seule caractérisée par des processus de durée très brève, générateurs de rayonnement VHF-UHF et clairement reliés aux discontinuités de propagation du leader négatif.

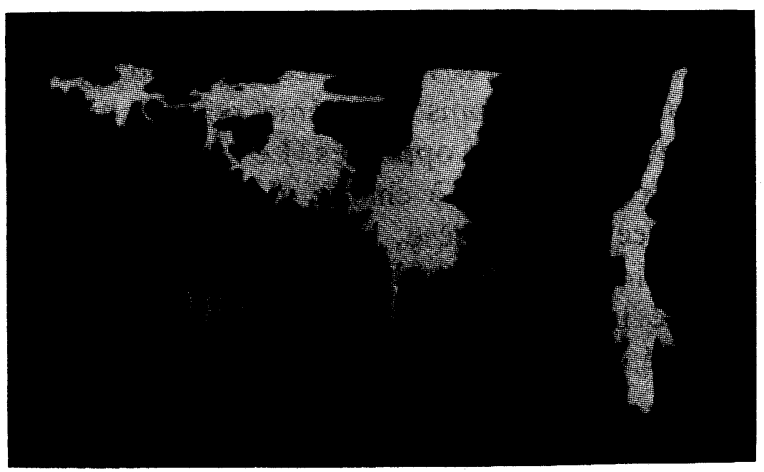

a)

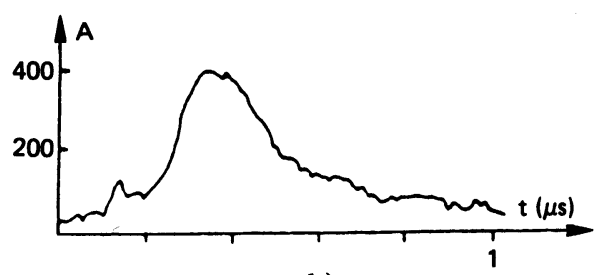

b)

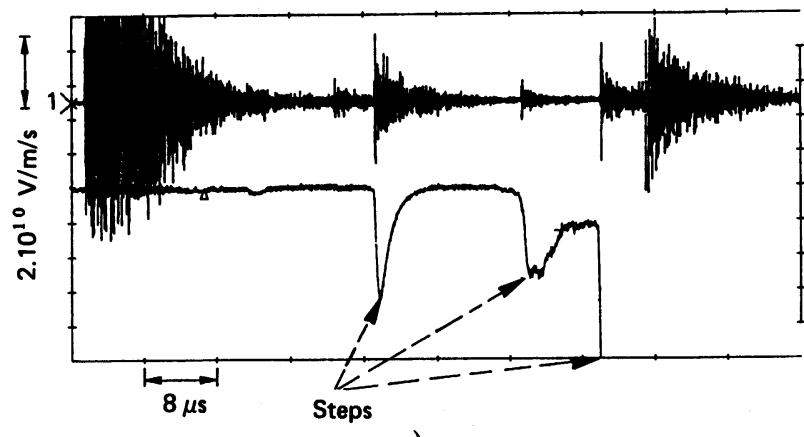

c)

Fig. 16. - (a) Caméragramme à balayage temporel montrant le développement par bonds (ou «steps ») d'un précurseur négatif (intervalle de $7 \mathrm{~m}$, tension: $2 \mathrm{MV}$ ) (d'après le Groupe des Renardières, 1981). (b) Courant associé à un «step ». (c) Enregistrement simultané de la dérivée du champ rayonné (haut) et du signal délivré par un photomultiplicateur, permettant d'identifier les steps. Le rayonnement observé pendant les 20 premières $\mu$ s est dû au générateur HT.

[(a) Streak cameragram of a negative stepped leader (gap length : $7 \mathrm{~m}$; voltage : $2 \mathrm{MV}$ ) (from les Renardières Group, 1981). (b) Step current. (c) Simultaneous recordings of the radiated $E$ field derivative (upper trace) and the photomultiplier signal allowing the steps detection. Radiation observed during the first $20 \mu \mathrm{s}$ is due to the HV generator triggering process.]

Pour interpréter les données interférométriques relatives au développement d'un éclair intranuage, nous nous appuierons, d'une part, sur les résultats obtenus en laboratoire et, d'autre part, sur la théorie du « leader bidirectionnel » proposée par Kasemir [7] et reprise par Mazur [12]. Selon cette théorie, le 
précurseur de l'éclair est en fait constitué de deux leaders, l'un positif et l'autre négatif, qui progressent simultanément, depuis un même point initial mais dans deux directions opposées. Le système constitué des deux leaders est à charge globale nulle.

Dans cette hypothèse, le développement du précurseur intervenant lors de la première phase de l'activité intranuage (Figs. 9a et 10a) serait en fait constitué de deux leaders dont seule la partie négative, discontinue, serait détectée en VHF-UHF, conformément aux résultats de laboratoire.

Cette hypothèse se trouve confirmée par les mesures de vitesse, de rythme d'émission et de périodicité spatiale ( 25 à $35 \mathrm{~m}$ ) qui constitue une mesure de la dimension d'un «step » du leader négatif intranuage, c'est-à-dire de la dimension du streamer de l'éclair naturel effectivement estimée par divers auteurs entre 10 et $50 \mathrm{~m}$ [1]. L'aspect diffus des sources observées par l'interféromètre est vraisemblablement lié au développement simultané des différentes branches constituant le leader négatif tandis que la progression du leader positif s'effectue sans émission détectable par l'interféromètre (Fig. 17a).

La géométrie des canaux observés pendant la seconde phase de l'éclair intranuage laisse supposer que la propagation des phénomènes dits de « recoil streamers " s'effectue selon les canaux du leader positif, en sens opposé à la propagation initiale de celui-ci. Cette hypothèse explique :

(i) les vitesses de propagation élevées associées au recoil streamers, difficilement explicables dans le cas d'une propagation hors d'un canal préexistant;

(ii) leur convergence vers la zone d'initiation de la phase leader.

L'observation des recoil streamers constituerait donc une visualisation indirecte des canaux du leader positif (Fig. 17b). Des mesures de champ électrique [21] ont montré que la charge totale neutralisée lors de la phase de recoil streamers était voisine de celle associée à la propagation de la phase leader. La seconde phase de l'activité intranuage pourrait donc correspondre à une neutralisation des charges laissées par le leader positif.

5.4 ConClusion. - La comparaison des mesures de rayonnement associé aux décharges de laboratoire et à l'éclair naturel permet donc de proposer une analyse qualitative des processus de développement de l'éclair intranuage. La mesure de l'amplitude du rayonnement (normalisée en distance et en bande passante) permet de proposer des facteurs d'échelles existant entre les deux types de décharges et constituant un apport indispensable à l'élaboration de modèles théoriques. Dans cette perspective, un modèle simple d'avancée du précurseur négatif a été proposé par Taudière [21]: cette modélisation repose sur une description de la décharge à partir

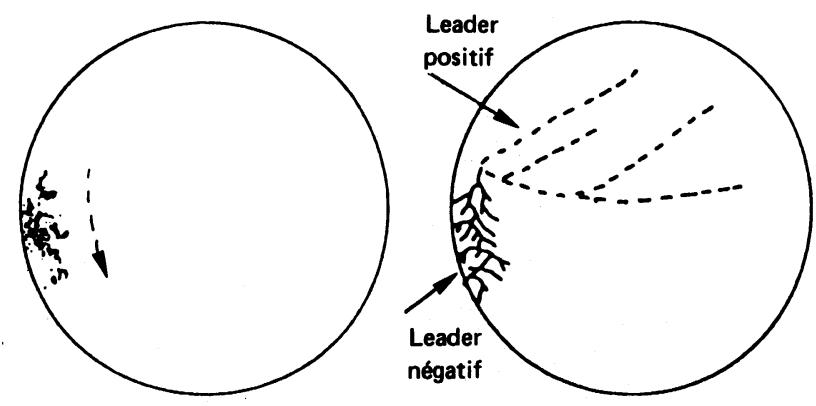

a)

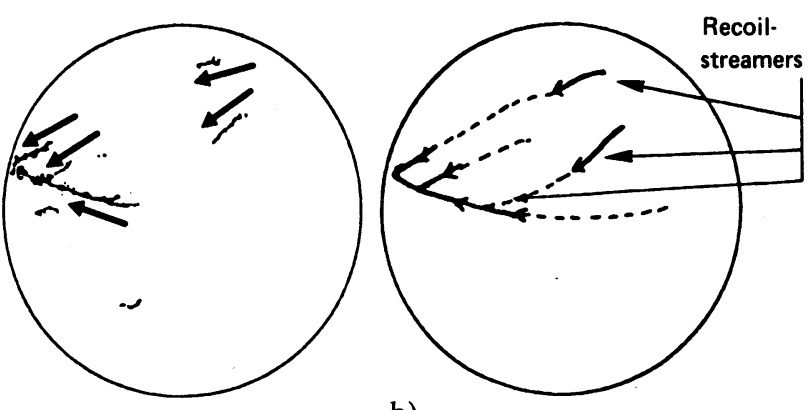

b)

Fig. 17. - (a) Première phase de développement d'un éclair intranuage. Localisation des sources VHF-UHF et schéma simplifié du phénomène de leader. (b) Deuxième phase du développement d'un éclair intranuage. Localisation des sources VHF-UHF et interprétation de l'organisation des sources.

[(a) First phase of an intracloud flash : location of the VHF-UHF sources and simplified scheme of the bidirectionnal leader development. (b) Second phase of the intracloud flash of figure 17a. Location of the VHF-UHF sources and interpretation the observed phenomena.]

d'un schéma électrique équivalent et a été confrontée de façon satisfaisante aux données disponibles tant dans le cas de la décharge de laboratoire que dans le cas du précurseur de l'éclair.

\section{Conclusion générale.}

Nous avons décrit un ensemble assez vaste d'investigations menées à partir de l'analyse du rayonnement VHF-UHF de l'éclair. Le champ d'application de telles études est large :

- définition de systèmes de détection des décharges orageuses : le système SAFIR, développé à l'ONERA, reposant sur un principe de localisation interférométrique, permet d'opérer une surveillance dans un rayon de $200 \mathrm{~km}$ environ autour du site à protéger ;

- approfondissement des études sur les cisaillements de vent au voisinage du sol : l'analyse de ce phénomène pourra être poursuivie à partir d'un suivi 
à grande échelle spatio-temporelle de l'activité électromagnétique ;

- enfin, l'analyse théorique et expérimentale des mécanismes de rayonnement à l'échelle de la décharge, confrontée à des études en laboratoire, aboutit à des descriptions phénoménologiques et à l'élaboration de modèles de certaines phases de l'éclair. Les connaissances actuelles en matière de foudroiement-avion engagent à poursuivre de tels travaux dans la perspective d'une modélisation du mécanisme d'interaction foudre-avion.

\section{Remerciements.}

Nous remercions le Laboratoire d'Essais Haute Tension de l'EDF, et plus particulièrement B. Hutzler et F. Morillon, pour l'ensemble des études menées sur les décharges de laboratoire. Nous tenons également à remercier le CNET-CRPE pour la réalisation et le traitement des mesures radar.

Les recherches présentées ici ont été financées par la Direction des Recherches, Etudes et Techniques.

\section{Bibliographie}

[1] Berger K., The earth flash, Lightning 1, Ed. R. H. Golde (Academic Press) 1977 pp. 119-190.

[2] Bondiou A., Labaune G. et Marque J. P., Electromagnetic radiation associated with the formation of an electric breakdown of air at atmospheric pressure, J. Appl. Phys. 61 (1987) 2.

[3] BRook M. et OgaWA T., The cloud discharge, Lightning 1, Ed. R. H. Golde (Academic Press) 1977.

[4] Groupe des Renardières, L'amorçage des grands intervalles d'air aux Renardières. Résultats de 1973, Electra 35 (1974). L'amorçage des grands intervalles d'air aux Renardières. Résultats de 1975, Electra 53 (1977). L'amorçage en polarité négative des grands intervalles d'air. Résultats de 1978, Electra 74 (1981).

[5] Hayenga C. O., Characteristics of lightning VHF radiation near the time of return stroke, $J$. Geophys. Res. 89 (D1) 1981.

[6] Hutzler B., Morillon F., Taudière, I., LABAUNE G. et Bondiou A., Two experimental studies on the laboratory long spark: VHF radiation and channel resistance evolution, Venise, 19-23 septembre 1988.

[7] KASEMIR H. W., Qualitative vebersicht neber potential. Feld und ladungverhaltnisse bei einer blitzentladung in der gewitterwolbre, Das Gewitter, (Hans Irael Akad. Verlangs. Ges. Geest und Portig K. G.) Leipzig, 1950.

[8] Labaune G., The role of the transient arc in the generation of metric and decimetric radiation by a lightning flash, Ann. Télécom. 39, 5-6 (1984).

[9] LARIGALDIE S., The spark propagation mechanisms in ambient air at the surface of a charged dielectric. I - Experimental : the main stages of the discharge, J. Appl. Phys. 61, 1 (1987).

[10] LARIGALDIE S., The spark propagation mechanisms in ambient air at the surface of a charged dielectric. II - Theoretical modelling, J. Appl. Phys. 61, 1 (1987).

[10bis] Leboulch M., Hamelin J., Rayonnement en ondes métriques et décimétriques des orages, Ann. Télécom. 40, 5-6, mai-juin 1985.

[11] LhermitTe R. et Williams E., Thunderstorm electrification. A case study, J. Geophys. Res. 90 (1985) 6071-6078.
[12] MAZUR V., Lightning initiation on aircraft in thunderstorms, 8th Int. Conf. on Atmospheric Electricity, Uppsala (Suède) juin 1988.

[13] Moreau J. P., Caractérisation du rayonnement électromagnétique des précurseurs de la foudre, Thèse de Doctorat d'Etat, Université Pierre et Marie Curie, décembre 1983.

[14] Pierce E. T., Atmospheric and Radio noise, Lightning, Ed. R. H. Golde (Academic Press Inc., London) 1977, Ch. 10.

[15] Proctor D. E., VHF radio picture of cloud flashes, J. Geophys. Res. 86 (1981) 4041-4071.

[16] Proctor D. E., UytenbogaARdt R. et Meredit V. M., VHF radio pictures of lightning flashes to ground, National Institute of Telecommunications Research, Johannesburg (1987).

[17] RichaRd P. et AuffRAy G., VHF-UHF interferometric measurements, applications to lightning discharge mapping, Radio Sci. 20 (1985) 171192.

[18] Richard P., Delannoy A., Labaune G. et LaroCHE P., Results of spatial and temporal characterization of the VHF-UHF radiation of lightning, J. Geophys. Res. 91 D1 (1986).

[19] Rustan P. L., UMAN M. A., Lightning source location from VHF radiation data for a flash at Kennedy Space Center, J. Geophys. Res. 85, C9 (1980).

[20] Taudière I., Bondiou A., Richard P., Labaune G., DelanNoy A., Quantative analysis of the VHF-UHF emission from lightining, 19th Int. Conf. on Lighting Protection, Graz (Autriche) Avril 1988.

[21] TAudière I., Analyse du rayonnement VHF-UHF associé à l'éclair. Application à un modèle d'avancée du précurseur négatif. Thèse de Doctorat, Université Paris VI, 12 octobre 1988.

[22] UMAN M. A. et KRIDER E. P., A review of natural lightning: experimental data and modelling, IEEE Transactions on Electromagnetic Compatibility, Vol. EMC-24, 2, mai 1982.

[23] Williams E., ORville R., Intracloud lightning as a precursor to thunderstorm microburst, ICOLSE 1988, Oklahoma (USA) 19-22 avril 1988 